\title{
The Modified Glasgow Prognostic Score in Patients with Gemcitabine-refractory Biliary Tract Cancer
}

\author{
NAOHIRO OKANO, AKIYOSHI KASUGA, KIRIO KAWAI, YOSHIYA YAMAUCHI, \\ TAKAAKI KOBAYASHI, DAISUKE NARUGE, FUMIO NAGASHIMA and JUNJI FURUSE \\ Department of Medical Oncology, Kyorin University Faculty of Medicine, Tokyo, Japan
}

\begin{abstract}
Background: No standard second-line chemotherapy has been yet established for gemcitabinerefractory biliary tract cancer (BTC). Patients and Methods: We conducted multivariable Cox regression analysis to examine the prognostic factors for overall survival (OS) in patients who had received gemcitabine-based treatment. Results: Forty-six patients received second-line chemotherapy. The median serum carbohydrate antigen 19-9 (CA 19-9) value was $487 \mathrm{U} / \mathrm{ml}$. The modified Glasgow prognostic score ( $m G P S)$ was: $0(n=24), 1(n=10)$, or 2 $(n=10)$. The second-line chemotherapy included: $S-1$ in 20 patients, gemcitabine-based in 20, and tyrosine kinase inhibitors in five. The median OS was 8.3 months, and the median progression-free survival was 3.0 months. Multivariate analysis identified serum CA 19-9 $\geq 500 \mathrm{U} / \mathrm{ml}$, $m G P S \geq 1$, and presence of liver metastasis as significant prognostic factors for OS. Conclusion: Second-line chemotherapy for gemcitabine-refractory BTC remains inadequate. Randomized trials with appropriate stratification criteria are required.
\end{abstract}

Gemcitabine plus cisplatin is widely recognized as the standard first-line chemotherapy for advanced biliary tract cancer (BTC) because randomized controlled trials have demonstrated an improved overall survival (OS) in patients receiving gemcitabine plus cisplatin compared to patients receiving gemcitabine monotherapy $(1,2)$. Although most patients develop tumor progression during gemcitabine plus cisplatin treatment, there are no published reports of randomized clinical trials of second-line chemotherapy in patients with gemcitabine-refractory BTC. Since S-1 has

Correspondence to: Naohiro Okano, MD, Department of Medical Oncology, Kyorin University Faculty of Medicine, 6-20-2, Shinkawa, Mitaka-shi, Tokyo, 181-8611, Japan. Tel: +81 422475511, Fax: +81 422440729, e-mail: naohiro-okano@ks.kyorin-u.ac.jp

Key Words: Biliary tract cancer, chemotherapy, gemcitabine, S-1, modified Glasgow prognostic score. been approved for use in patients with BTC, S-1 has been commonly used for second-line chemotherapy in patients with gemcitabine-refractory BTC in Japan. To date, the usefulness of S-1 has been evaluated in two single-arm clinical trials. In one, the response rate (RR), median progression-free survival (PFS) and median OS were 22.7\%, 5.4 months and 13.5 months, respectively (3). In the other, the RR, median PFS and median OS were 7.5\%, 2.5 months and 6.8 months, respectively (4). Thus, the efficacy of S-1 as second-line chemotherapy differed between the two clinical trials. We speculated that the differences in the sample size and patient backgrounds, such as history of surgery, were responsible for the difference in efficacy between the two trials. Therefore, no standard chemotherapy has been established yet for patients with gemcitabinerefractory BTC.

The modified Glasgow prognostic score (mGPS) is based on the serum C-reactive protein (CRP) and serum albumin levels, and is an inflammation-based prognostic factor (5). It has been recognized as a prognostic factor in patients with various types of solid tumors $(6,7)$. To the best of our knowledge, there is no report on the relationship between mGPS and OS in patients with gemcitabine-refractory BTC receiving second-line chemotherapy.

Therefore, we carried out a retrospective evaluation of the efficacy of second-line chemotherapy and the prognostic factors for OS in patients with gemcitabine-refractory BTC receiving second-line chemotherapy. Furthermore, we investigated the relationship between the mGPS and OS in the setting of second-line chemotherapy.

\section{Patients and Methods}

Study population. We retrospectively examined the data of patients with advanced BTC who had received first-line treatment with a gemcitabine-based regimen between January 2009 and December 2015 at Kyorin University Hospital. Patients with histologically confirmed unresectable or recurrent BTC, including intrahepatic bile duct, extrahepatic bile duct, gallbladder and ampulla of Vater cancer, who had received first-line chemotherapy were reviewed. Among 102 patients who had received a gemcitabine-based regimen as first- 
GEM-based treatment $(n=102)$

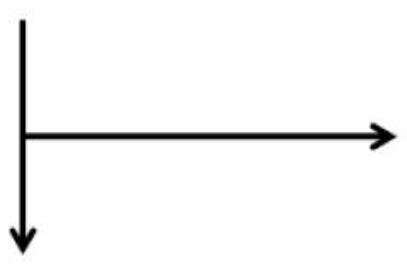

No indication for second-line treatment $(n=28)$

Patient refusal $(n=8)$

Unsuccessful biliary drainage $(n=7)$

Lost to follow-up $(n=6)$

Continuation of treatment $(n=4)$

Complete remission $(n=2)$

Treatment-related death $(n=1)$

\section{Second-line treatment $(n=46)$}

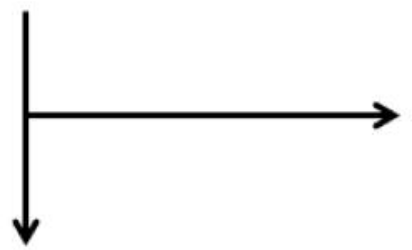

Treatment at another hospital $(n=1)$

Eligible for this study $(n=45)$

Figure 1. Consort diagram. Among 102 patients treated with a gemcitabine (gemcitabine)-based regimen as first-line treatment, 46 patients (45.1\%) received second-line chemotherapy.

line treatment, 46 patients $(45 \%)$ also received second-line chemotherapy (Figure 1). We analyzed the incidence of adverse events (AEs), the antitumor effect of the treatment, PFS, OS and prognostic factors associated with OS in the patients who were receiving second-line chemotherapy. One patient was excluded from this study because the second-line chemotherapy was initiated at another hospital. This study was conducted with the approval of the local Institutional Review Board (approval number: 795).

Assessment of response and AEs. The tumor response was evaluated by computed tomography (CT) or magnetic resonance imaging (MRI), in accordance with the Response Evaluation Criteria in Solid Tumors 1.1. CT and MRI were basically performed every 2 to 3 months until confirmation of progressive disease. Adverse events were recorded in accordance with the Common Terminology Criteria for Adverse Events 4.0.

Analysis of prognostic factors. All data were collected before the second-line chemotherapy was initiated. The mGPS was calculated using the serum CRP and serum albumin as follows: Patients with a normal serum CRP level were assigned a score of 0 , patients with both elevated serum CRP $(\geq 1.0 \mathrm{mg} / \mathrm{dl})$ and reduced serum albumin level $(<3.5 \mathrm{~g} / \mathrm{dl})$ were assigned a score of 2 , patients with only elevated serum CRP level were assigned a score of 1 . Factors potentially influencing OS were selected as follows: age $(<70 \mathrm{vs}$. $\geq 70$ years), gender, Eastern Cooperative Oncology Group performance status (ECOG PS) (0vs. 1), history/no history of prior surgical resection, PFS after first-line treatment ( $<6$ months $v s . \geq 6$ months), primary tumor site (non-gallbladder $v s$. gallbladder), presence/absence of liver metastasis, lung metastasis and/or peritoneal dissemination, serum alkaline phosphatase (ALP) level ( $<400 \mathrm{IU} / 1 v s . \geq 400 \mathrm{IU} / 1$ ), serum carbohydrate antigen 19-9 (CA199) level $(<500 \mathrm{U} / \mathrm{ml} v s$. $\geq 500 \mathrm{U} / \mathrm{ml})$, mGPS (0 vs. 1 or 2$)$, and chemotherapy regimen (monotherapy $v s$. combination therapy). The patients were divided into two groups based on levels higher and lower than the median age, ALP and CA19-9 level.

Statistical methods. PFS and OS were calculated using the KaplanMeier method and differences were evaluated using the log-rank test. Univariate analysis was first performed to identify the prognostic factors for OS in the patients receiving second-line chemotherapy. Factors identified by univariate analysis as being statistically significant $(p<0.05)$ were entered into Cox proportional hazard regression model using a stepwise procedure for multivariate analysis. The statistical analyses were performed by the statistical software package SPSS version 22.0 for Windows (IBM Corp., Armonk, NY, USA).

\section{Results}

Patients characteristics. The patients characteristics are shown in Table I. The mGPS could not be calculated for one patient because serum albumin data were missing. A total of 102 patients received gemcitabine-based treatment between January 2009 and December 2015 at our hospital. Of these, 
Table I. Patients characteristics.

\begin{tabular}{lc}
\hline Characteristic & $\mathrm{n}=45$ \\
\hline Median age (range), years & $68(35-84)$ \\
Gender, $\mathrm{n}(\%)$ & \\
Male/Female & $25(56) / 20(44)$ \\
ECOG PS, n (\%) & $25(56) / 20(44)$ \\
0/1 & \\
Primary tumor site, $\mathrm{n}(\%)$ & $18(40)$ \\
Gallbladder & $15(33)$ \\
Extrahepatic bile duct & $10(22)$ \\
Intrahepatic bile duct & $2(5)$ \\
Ampulla of vater & $27(60)$ \\
Extent of disease, $\mathrm{n}(\%)$ & $15(33)$ \\
Metastatic & $3(7)$ \\
Recurrent & $405(109-1619)$ \\
Locally advanced & $0.5(0-8.1)$ \\
Median ALP (range), IU/l & $3.6(2.5-4.7)$ \\
Median CRP (range), mg/dl & $487(<2.0-120000)$ \\
Median Alb (range), g/dl & \\
Median CA19-9 (range), U/ml & 24 \\
mGPS & 10 \\
0 & 10 \\
1 & $19(42)$ \\
2 & $7(16)$ \\
First-line treatment, $\mathrm{n}(\%)$ & $4(9)$ \\
Gem +cisplatin & $1(2)$ \\
Gem +S-1 & \\
Gem monotherapy & \\
Gem +elpamotide & \\
Gem +radiation & \\
\hline
\end{tabular}

ECOG PS, Eastern Cooperative Oncology Group performance status; ALP, serum alkaline phosphatase; CRP, serum C-reactive protein; Alb, serum albumin; CA19-9, serum carbohydrate antigen 19-9; mGPS, modified Glasgow prognostic score; Gem, gemcitabine.

28 patients $(27 \%)$ were judged as not being suitable candidates for second-line chemotherapy because of poor general physical conditions. Eight patients $(8 \%)$ wished to receive only palliative care despite being considered as suitable for receiving second-line chemotherapy. Seven patients $(7 \%)$ could not receive second-line chemotherapy because of obstructive jaundice as a result of unsuccessful biliary drainage. Complete response (CR) was achieved in two patients $(2 \%)$, pathological CR was achieved in one patient after 2 years of intensive combined chemotherapy with gemcitabine and S-1 (8). There was one (1\%) treatmentrelated death due to intestinal pneumonia induced by gemcitabine. Thus, 46 (45\%) patients with gemcitabinerefractory BTC who received second-line chemotherapy were finally eligible for this study. However, we excluded one patient because his treatment had been started at another hospital, and finally included 45 patients in this study (Figure 1).
Table II. Second-line chemotherapy $(n=45)$.

\begin{tabular}{lc}
\hline & $\mathrm{n}(\%)$ \\
\hline S-1 & $20(44)$ \\
Gem +oxaliplatin & $6(13)$ \\
Gem +cisplatin & $5(11)$ \\
Gem monotherapy & $5(11)$ \\
Tyrosine kinase inhibitors & $5(11)$ \\
$\quad$ Axitinib & 2 \\
Lenvatinib & 2 \\
Trametinib & 1 \\
Gem+S-1 & $2(5)$ \\
Fixed-dose Gem+S-1 & $2(5)$ \\
\hline
\end{tabular}

Gem, Gemcitabine.

Second-line chemotherapy. The second-line chemotherapy regimens used are listed in Table II. Forty-five patients received second-line chemotherapy. S-1 used as the standard chemotherapy and as part of a clinical trial of patients with gemcitabine-refractory BTC. Gemcitabine plus oxaliplatin and tyrosine kinase inhibitors selected as part of a clinical trial in some patients. Gemcitabine plus cisplatin was selected in patients with BTC refractory to gemcitabine plus S-1. Gemcitabine monotherapy was selected in patients in whom the second drug had to be discontinued because of the emergence of AEs in response to combination therapies such as gemcitabine plus cisplatin or gemcitabine plus $\mathrm{S}-1$. Gemcitabine plus $\mathrm{S}-1$ is selected in clinical practice in patients with BTC refractory to gemcitabine plus cisplatin. Fixed-dose gemcitabine plus S-1 was selected by the physicians because this combination as first-line chemotherapy has been shown to be highly effective: The patient had a target lesion in lymph node before first-line chemotherapy. The target lesion was a maximum tumor size reduction of $75 \%$ and PFS was 10.3 months.

Efficacy and safety. There were no patients with CR or PR. The tumor response was classified as stable disease in 26 $(57.8 \%)$ patients and as progressive disease in 37 (37.8\%) patients. Antitumor effect was not evaluated in two patients because these patients were shifted to palliative care because they developed biliary tract infection immediately after the start of chemotherapy. The RR was $0 \%$ and the disease control rate was $57.8 \%$ for second-line chemotherapy in patients with gemcitabine-refractory BTC.

The PFS and OS curves are shown Figure 2. The median PFS and OS were 3.0 months [95\% confidence interval $(\mathrm{CI})=1.4-4.5$ months] and 8.3 months $(95 \% \mathrm{CI}=4.7-11.9$ months), respectively.

The most commonly encountered grade 3 or 4 AEs were bone marrow suppression and biliary tract infection. In regard to hematological grade 3 or 4 AEs, neutropenia, anemia and leukopenia were observed in five (11\%), five 


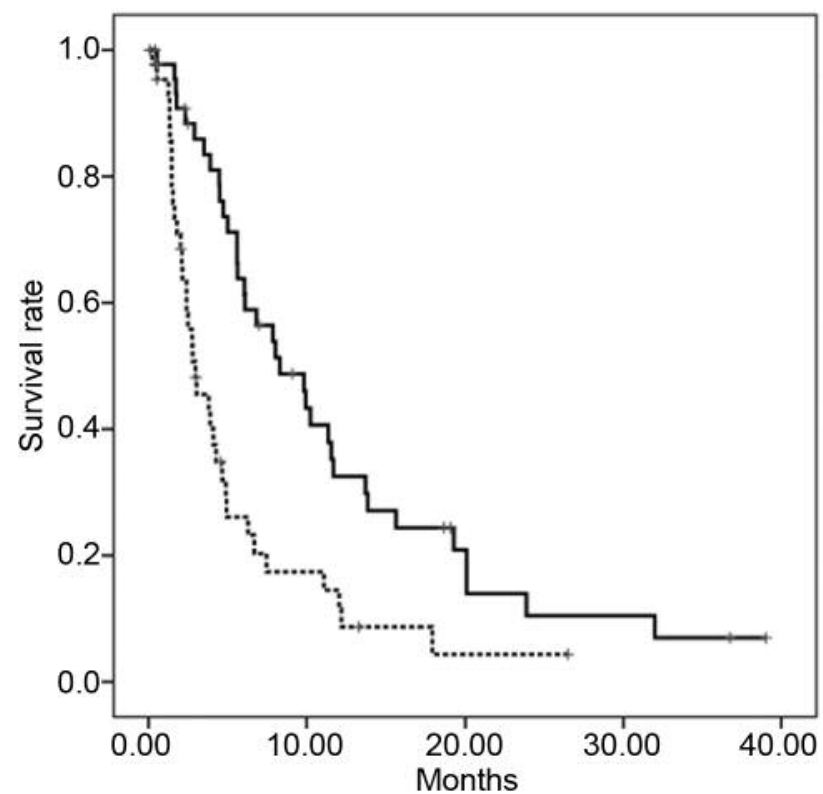

Figure 2. Kaplan-Meier curves for overall survival (black line) and progression-free survival (dotted line). The median progression-free survival and overall survival were 3.0 (95\% confidence interval $=1.4$ 4.5) months and 8.3 (95\% confidence interval=4.7-11.9) months, respectively.

(11\%), and one (2\%) patient, respectively. In regard to nonhematological grade 3 or 4 AEs, biliary tract infection, anorexia, thromboembolic events, hand-foot syndrome, hypertension, and creatine phosphokinase increased were observed in six $(13 \%)$, one $(2 \%)$, one $(2 \%)$, one $(2 \%)$, one $(2 \%)$, and one (2\%) patient, respectively. There was one treatment-related death due to biliary tract infection.

Univariate and multivariate analysis to identify factors affecting OS. In the univariate analysis, ECOG PS, PFS after first-line treatment, liver metastasis, ALP level, CA 19-9 level and mGPS were identified as prognostic factors for OS (Table III). The variables that were identified by univariate analysis were entered into the multivariate analysis model. Multivariate analysis identified raised serum CA 19-9 level, mGPS $>0$ and presence of liver metastasis as an independent prognostic factors for reduced OS (Table IV).

Association of the mGPS with OS. The relationship between the mGPS and OS is shown Figure 3. The median OS values were 11.7 months (95\% CI=6.2-17.2 months), 9.8 months (95\% CI=0.7-18.9 months) and 4.5 months (95\% CI, 1.2-7.7 months) in patients with mGPS 0,1 , and 2 , respectively. The log-rank test showed significant differences $(p=0.01)$ among the three groups.

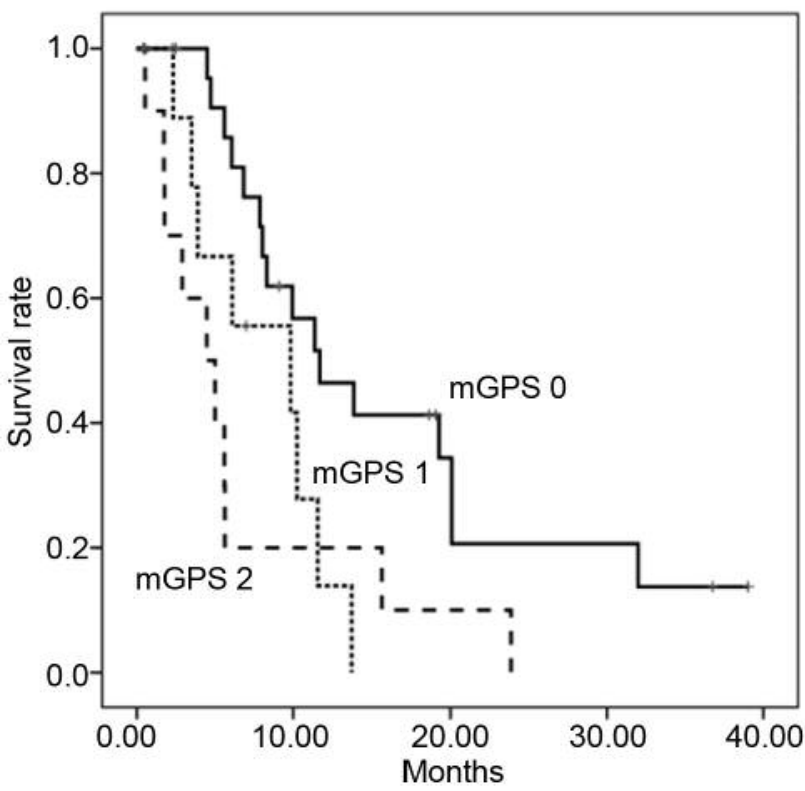

Figure 3. Kaplan-Meier curves for overall survival according to the modified Glasgow prognostic factor ( $m G P S$ ). The median overall survival was 11.7 (95\% confidence interval=6.2-17.2) months, 9.8 (95\% confidence interval=0.7-18.9) months and 4.5 (95\% confidence interval $=1.2-7.7)$ months in the patient groups with $m G P S 0,1$ and 2, respectively. The logrank test showed significant differences among the groups $(p=0.01)$.

\section{Discussion}

This retrospective study conducted on patients with gemcitabine-refractory BTC receiving second-line chemotherapy showed a RR of $0 \%$, DCR of $57.8 \%$, median PFS of 3.0 months, and median OS of 8.3 months. Furthermore, mGPS was identified as an independent prognostic factor for OS in patients with gemcitabinerefractory BTC receiving second-line chemotherapy, and the OS was found to be dependent on the mGPS.

Although gemcitabine plus cisplatin has been used as effective first-line chemotherapy, most patients show tumor progression over time. However, there are no established second-line chemotherapies for patients with gemcitabinerefractory BTC. To date, some single-arm trials have shown modest efficacy of second-line chemotherapy, with a median OS and PFS of 4.1 to 13.5 months and 1.6 to 5.4 months, respectively $(3,4,9-17)$. Our results were similar to previous reports of the efficacy of second-line chemotherapy in patients with advanced BTC. The secondline treatments used to date do not seem to have sufficient antitumor efficacy.

The current study demonstrated a transition rate from firstline treatment to second-line chemotherapy of $45 \%$. Even though patients receiving gemcitabine-based first-line 
Table III. Univariate analysis to identify prognostic factors for overall survival (OS) in patients receiving second-line chemotherapy.

\begin{tabular}{|c|c|c|c|c|}
\hline Variable & $\mathrm{n}$ & $\begin{array}{c}\text { Median OS } \\
\text { (months) }\end{array}$ & $\begin{array}{c}\text { Hazard ratio } \\
(95 \% \mathrm{CI})\end{array}$ & $p$-Value \\
\hline \multicolumn{5}{|l|}{ Age } \\
\hline$<70$ Years & 24 & 9.8 & 1 & \multirow{2}{*}{0.79} \\
\hline$\geq 70$ Years & 21 & 8.0 & $0.91(0.47-1.79)$ & \\
\hline \multicolumn{5}{|l|}{ Gender } \\
\hline Female & 20 & 9.9 & 1 & \multirow{2}{*}{0.52} \\
\hline Male & 25 & 7.9 & $1.25(0.63-2.47)$ & \\
\hline \multicolumn{5}{|l|}{ ECOG PS } \\
\hline 0 & 25 & 11.4 & 1 & \multirow{2}{*}{0.03} \\
\hline 1 & 20 & 5.6 & $2.20(1.10-4.42)$ & \\
\hline \multicolumn{5}{|c|}{ Prior surgical resection } \\
\hline Yes & 15 & 11.4 & 1 & \multirow{2}{*}{0.27} \\
\hline No & 30 & 8.0 & $1.49(0.73-3.02)$ & \\
\hline \multicolumn{5}{|c|}{ PFS after 1st line therapy } \\
\hline$\geq 6$ months & 21 & 13.7 & 1 & \multirow{2}{*}{0.01} \\
\hline$<6$ months & 24 & 5.6 & $2.42(1.22-4.82)$ & \\
\hline \multicolumn{5}{|l|}{ Primary tumor site } \\
\hline Non-gallbladder & 27 & 8.0 & 1 & \multirow{2}{*}{0.81} \\
\hline Gallbladder & 18 & 11.7 & $0.92(0.47-1.81)$ & \\
\hline \multicolumn{5}{|l|}{ Liver metastasis } \\
\hline Absent & 19 & 13.9 & 1 & \multirow{2}{*}{$<0.01$} \\
\hline Present & 26 & 5.6 & $3.86(1.70-8.80)$ & \\
\hline \multicolumn{5}{|c|}{ Peritoneal dissemination } \\
\hline Absent & 35 & 8.0 & 1 & \multirow{2}{*}{0.67} \\
\hline Present & 10 & 8.3 & $0.85(0.39-1.83)$ & \\
\hline \multicolumn{5}{|l|}{ Lung metastasis } \\
\hline Absent & 35 & 8.3 & 1 & \multirow{2}{*}{0.99} \\
\hline Present & 10 & 6.1 & $0.99(0.46-2.15)$ & \\
\hline \multicolumn{5}{|l|}{ ALP } \\
\hline$<400 \mathrm{IU} / 1$ & 22 & 13.7 & 1 & \multirow{2}{*}{$<0.01$} \\
\hline$\geq 400 \mathrm{IU} / 1$ & 23 & 6.1 & $2.65(1.31-5.36)$ & \\
\hline \multicolumn{5}{|l|}{ CA19-9 } \\
\hline$<500 \mathrm{U} / \mathrm{ml}$ & 23 & 11.7 & 1 & \multirow{2}{*}{$<0.01$} \\
\hline$\geq 500 \mathrm{U} / \mathrm{ml}$ & 22 & 5.6 & $2.72(1.34-5.52)$ & \\
\hline \multicolumn{5}{|l|}{ mGPS } \\
\hline 0 & 24 & 11.7 & 1 & \multirow{2}{*}{$<0.01$} \\
\hline 1 or 2 & 20 & 5.6 & $2.74(1.36-5.53)$ & \\
\hline \multicolumn{5}{|l|}{ Monotherapy } \\
\hline Yes & 30 & 9.9 & 1 & \multirow{2}{*}{0.13} \\
\hline No & 15 & 8.0 & $1.70(0.85-3.40)$ & \\
\hline
\end{tabular}

ECOG PS, Eastern Cooperative Oncology Group performance status; PFS, progression-free survival; ALP, serum alkaline phosphatase; CA19-9, serum carbohydrate antigen 19-9; mGPS, modified Glasgow prognostic score; OS, overall survival; CI, confidence interval.

treatment eventually develop tumor progression, about $50 \%$ of these patients with gemcitabine-refractory BTC need an effective second-line chemotherapy. Thus, establishment of second-line chemotherapy for patients with advanced BTC is urgently required.

Analyses of prognostic factors identified high serum CA19-9 level, a high mGPS score and the presence of liver metastasis as independent poor prognostic factors. Previous
Table IV. Multivariate analysis to identify prognostic factors for overall survival in patients receiving second-line chemotherapy.

\begin{tabular}{lccc}
\hline Variable & Hazard ratio & $95 \% \mathrm{CI}$ & $p$-Value \\
\hline CA19-9 & 1 & & \\
$\quad<500 \mathrm{U} / \mathrm{ml}$ & 3.45 & $1.52-7.85$ & 0.003 \\
$\quad 5500 \mathrm{U} / \mathrm{ml}$ & 1 & & \\
$\mathrm{mGPS}$ & 3.05 & $1.40-6.67$ & 0.005 \\
$\quad \begin{array}{l}1-2 \\
\text { Liver metastasis }\end{array}$ & 1 & & \\
$\quad \begin{array}{l}\text { Absent } \\
\text { Present }\end{array}$ & 2.62 & $1.01-6.80$ & 0.048 \\
\hline
\end{tabular}

CA19-9, Serum carbohydrate antigen 19-9; mGPS, modified Glasgow prognostic score; CI, confidence interval.

studies have reported presence of ascites, lack of response to first-line chemotherapy, elevated serum CA19-9, poor PS, no history of surgery for the primary tumor, intrahepatic bile duct cancer, and presence of metastatic disease as poor prognostic factors for OS in patients with advanced BTC receiving second-line chemotherapy (1821). Although inflammation-based prognostic factors, such as the mGPS, have been demonstrated as prognostic factors in patients with BTC receiving first-line chemotherapy or undergoing surgical resection (22-28), there have been no reports yet of identification of such factors as having an influence on the prognosis in patients with gemcitabinerefractory BTC receiving second-line chemotherapy. The results of our current study suggest that inflammation-based prognostic factors are important in patients with BTC receiving second-line chemotherapy, just as in patients receiving first-line chemotherapy or undergoing surgical resection.

This study had several limitations. Firstly, it was retrospective in its nature, the sample size was small and it was a single-center study. Secondly, it included patients treated before gemcitabine plus cisplatin was approved in Japan. Finally, various second-line chemotherapy regimens, including clinical trial regimens, were used. Therefore, a prospective multi-center study is needed to confirm the conclusions of this study in regard to these prognostic factors.

In conclusion, second-line chemotherapy using existing drugs such as S-1 has only modest activity against gemcitabine-refractory BTC. Randomized controlled trials using newer agents and appropriate stratification criteria for the patients, including serum CA19-9, mGPS, and presence/ absence of liver metastasis, are required to establish a standard second-line chemotherapy for gemcitabinerefractory BTC. 


\section{References}

1 Valle J, Wasan H, Palmer DH, Cunningham D, Anthoney A, Maraveyas A, Madhusudan S, Iveson T, Hughes S, Pereira SP, Roughton $\mathrm{M}$ and Bridgewater $\mathrm{J}$ : Cisplatin plus gemcitabine versus gemcitabine for biliary tract cancer. N Engl J Med 362: 1273-1281, 2010.

2 Okusaka T, Nakachi K, Fukutomi A, Mizuno N, Ohkawa S, Funakoshi A, Nagino M, Kondo S, Nagaoka S, Funai J, Koshiji M, Nambu Y, Furuse J, Miyazaki M and Nimura Y: Gemcitabine alone or in combination with cisplatin in patients with biliary tract cancer: A comparative multicentre study in Japan. Br J Cancer 103: 469-474, 2010.

3 Sasaki T, Isayama H, Nakai Y, Mizuno S, Yamamoto K, Yagioka H, Yashima Y, Kawakubo K, Kogure H, Togawa O, Matsubara S, Ito Y, Sasahira N, Hirano K, Tsujino T, Toda N, Tada M, Omata $\mathrm{M}$ and Koike $\mathrm{K}$ : Multicenter phase II study of S-1 monotherapy as second-line chemotherapy for advanced biliary tract cancer refractory to gemcitabine. Invest New Drugs 30: 708-713, 2012.

4 Suzuki E, Ikeda M, Okusaka T, Nakamori S, Ohkawa S, Nagakawa T, Boku N, Yanagimoto H, Sato T and Furuse J: A multicenter phase II study of S-1 for gemcitabine-refractory biliary tract cancer. Cancer Chemother Pharmacol 71: 11411146, 2013.

5 Forrest LM, McMillan DC, McArdle CS, Angerson WJ and Duniop DJ: Evaluation of cumulative prognostic scores based on the systemic inflammatory response in patients with inoperable non-small-cell lung cancer. Br J Cancer 89: 10281030, 2003.

6 McMillan DC: The systemic inflammation-based Glasgow Prognostic Score: A decade of experience in patients with cancer. Cancer Treatment Reviews 39: 534-540, 2013.

7 Proctor MJ, Horgan PG, Talwar D, Fletcher CD, Morrison DS and McMillan DC: Optimization of the systemic inflammationbased Glasgow Prognostic Score: A Glasgow Inflammation Outcome Study. Cancer 119: 2325-2332, 2013.

8 Watanabe T, Furuse J, Okano N, Suzuki Y, Kamma H and Sugiyama M: A pathological complete response after combined chemotherapy of gemcitabine and S-1 in advanced biliary tract cancer with para-aortic lymph nodes metastasis: a case report. Surg Case Rep 3: 26, 2017.

9 Bengala C, Bertolini F, Malavasi N, Boni C, Aitini E, Dealis C, Zironi S, Depenni R, Fontana A, Del Giovane C, Luppi G and Conte P: Sorafenib in patients with advanced biliary tract carcinoma: A phase II trial. Br J Cancer 102: 68-72, 2010.

10 Bekaii-Saab T, Phelps MA, Li X, Saji M, Goff L, Kauh JS, O'Neil BH, Balsom S, Balint C, Liersemann R, Vasko VV, Bloomston M, Marsh W, Doyle LA, Ellison G, Grever M, Ringel MD and Villalona-Calero MA: Multi-institutional phase II study of selumetinib in patients with metastatic biliary cancers. J Clin Oncol 29: 2357-2363, 2011.

11 Oh SY, Jeong CY, Hong SC, Kim TH, Ha CY, Kim HJ, Lee GW, Hwang IG, Jang JS, Kwon HC and Kang JH: Phase II study of second line gemcitabine single chemotherapy for biliary tract cancer patients with 5-fluorouracil refractoriness. Invest New Drugs 29: 1066-1072, 2011.

12 Sasaki T, Isayama H, Nakai Y, Mizuno S, Yamamoto K, Yagioka H, Yashima Y, Kawakubo K, Kogure H, Togawa O, Matsubara S, Sasahira N, Hirano K, Tsujino T, Tada M, Omata M and
Koike K: Feasibility study of gemcitabine and cisplatin combination chemotherapy for patients with refractory biliary tract cancer. Invest New Drugs 29: 1488-1493, 2011.

13 Yi JH, Thongprasert S, Lee J, Doval DC, Park SH, Park JO, Park YS, Kang WK and Lim HY: A phase II study of sunitinib as a second-line treatment in advanced biliary tract carcinoma: A multicentre, multinational study. Eur J Cancer 48: 196-201, 2012.

14 Sasaki T, Isayama H, Nakai Y, Takahara N, Satoh Y, Takai D, Kogure H, Yamamoto N, Hirano K, Tada M, Yatomi Y and Koike $\mathrm{K}$ : A pilot study of salvage irinotecan monotherapy for advanced biliary tract cancer. Anticancer Res 33: 2619-2622, 2013.

15 Moretto R, Raimondo L, De Stefano A, Cella CA, Matano E, De Placido $S$ and Carlomagno C: FOLFIRI in patients with locally advanced or metastatic pancreatic or biliary tract carcinoma: a monoinstitutional experience. Anticancer Drugs 24: 980-985, 2013.

16 He S, Shen J, Sun X, Liu L and Dong J: A phase II FOLFOX-4 regimen as second-line treatment in advanced biliary tract cancer refractory to gemcitabine/cisplatin. J Chemother 26: 243-247, 2014.

17 Goyal L, Zheng H, Yurgelun MB, Abrams TA, Allen JN, Cleary JM, Knowles M, Regan E, Reardon A, Khachatryan A, Jain RK, Nardi V, Borger DR, Duda DG and Zhu AX: A phase 2 and biomarker study of cabozantinib in patients with advanced cholangiocarcinoma. Cancer 123: 1979-1988, 2017.

18 Kobayashi S, Ueno M, Ohkawa S, Andou T, Kameda R, Yamamoto $\mathrm{N}$ and Morinaga $\mathrm{S}$ : A retrospective study of $\mathrm{S}-1$ monotherapy as second-line treatment for patients with advanced biliary tract cancer. Jpn J Clin Oncol 42: 800-806, 2012.

19 Fornaro L, Cereda S, Aprile G, Di Girolamo S, Santini D, Silvestris N, Lonardi S, Leone F, Milella M, Vivaldi C, Belli C, Bergamo F, Lutrino SE, Filippi R, Russano M, Vaccaro V, Brunetti A, Rotella V, Falcone A, Barbera MA, Corbelli J, Fasola G, Aglietta M, Zagonel V, Reni M, Vasile E and Brandi G: Multivariate prognostic factors analysis for second-line chemotherapy in advanced biliary tract cancer. Br J Cancer 110: 2165-2169, 2014.

20 Brieau B, Dahan L, De Rycke Y, Boussaha T, Vasseur P, Tougeron D, Lecomte T, Coriat R, Bachet JB, Claudez P, Zaanan A, Soibinet P, Desrame J, Thirot-Bidault A, Trouilloud I, Mary F, Marthey L, Taieb J, Cacheux W and Lièvre A: Second-line chemotherapy for advanced biliary tract cancer after failure of the gemcitabine-platinum combination: A large multicenter study by the Association des Gastro-Enterologues Oncologues. Cancer 121: 3290-3297, 2015.

21 Kim BJ, Yoo C, Kim KP, Hyung J, Park SJ, Ryoo BY and Chang HM: Efficacy of fluoropyrimidine-based chemotherapy in patients with advanced biliary tract cancer after failure of gemcitabine plus cisplatin: retrospective analysis of 321 patients. Br J Cancer 116: 561-567, 2017.

22 Oshiro Y, Sasaki R, Fukunaga K, Kondo T, Oda T, Takahashi H and Ohkohchi N: Inflammation-based prognostic score is a useful predictor of postoperative outcome in patients with extrahepatic cholangiocarcinoma. J Hepatobiliary Pancreat Sci 20: 389-395, 2013.

23 Shiba H, Misawa T, Fujiwara Y, Futagawa Y, Furukawa K, Haruki K, Iwase R, Wakiyama S, Ishida $Y$ and Yanaga $K$ : Glasgow prognostic score predicts therapeutic outcome after pancreaticoduodenectomy for carcinoma of the ampulla of vater. Anticancer Res 33: 2715-2721, 2013. 
24 Iwaku A, Kinoshita A, Onoda H, Fushiya N, Nishino H, Matsushima $M$ and Tajiri H: The Glasgow Prognostic Score accurately predicts survival in patients with biliary tract cancer not indicated for surgical resection. Med Oncol 31: 787, 2014.

25 Moriwaki T, Ishige K, Araki M, Yoshida S, Nishi M, Sato M, Yamada T, Yamamoto Y, Ozeki M, Ishida H, Yamaguchi T, Matsuda K, Murashita T, Abei M and Hyodo I: Glasgow Prognostic Score predicts poor prognosis among advanced biliary tract cancer patients with good performance status. Med Oncol 31: 287, 2014

26 Wu XS, Shi LB, Li ML, Ding Q, Weng H, Wu WG, Cao Y, Bao RF, Shu YJ, Ding QC, Mu JS, Gu J, Dong P and Liu YB: Evaluation of two inflammation-based prognostic scores in patients with resectable gallbladder carcinoma. Ann Surg Oncol 21: 449-457, 2014.
27 Shiba H, Misawa T, Fujiwara Y, Futagawa Y, Furukawa K, Haruki K, Iwase R, Iida T and Yanaga K: Glasgow prognostic score predicts outcome after surgical resection of gallbladder cancer. World J Surg 39: 753-758, 2015.

28 Pan QX, Su ZJ, Zhang JH, Wang CR and Ke SY: Glasgow Prognostic Score predicts prognosis of intrahepatic cholangiocarcinoma. Mol Clin Oncol 6: 566-574, 2017.

Received November 30, 2017

Revised January 9, 2018

Accepted January 11, 2018 